\section{P170 ANTIMALARIAL AGENT USE IS ASSOCIATED WITH FAVOURABLE PHYSICAL FUNCTIONING IN PATIENTS WITH SYSTEMIC LUPUS ERYTHEMATOSUS}

Ioannis Parodis, Sofia Soukka, Alvaro Gomez, Yvonne Enman, Petter Johansson, Sharzad Emamikia, Katerina Chatzidionysiou. Division of Rheumatology, Dept. of Medicine, Karolinska Institutet and Rheumatology, Karolinska University Hospital, Stockholm, Sweden

\subsection{6/lupus-2020-eurolupus.212}

Background Patients with Systemic Lupus Erythematosus (SLE) suffer an impaired health-related quality of life (HRQoL), and the majority of them experience fatigue as a major problem. Traditionally, treatment of SLE has been symptomatic, and antimalarial agents (AMA) are considered a cornerstone of SLE treatment. In previous literature, results regarding the effect of AMA on HRQoL have been conflicting. In this study, we aimed at investigating the potential influence of AMA on SLE patients' self-perception of HRQoL aspects.

Methods We utilised pooled baseline data from the BLISS-52 and BLISS-76 clinical trials of belimumab $(\mathrm{N}=1684)$. Access to data was granted by GlaxoSmithKline. The patients' HRQoL and fatigue were self-reported using the Medical Outcomes Study short form 36 (SF-36) health survey, the functional assessment of chronic illness therapy (FACIT)-Fatigue scale and the three-level EuroQol 5 Dimension (EQ-5D) questionnaire. Minimal clinically important difference (MCID) was set to $\geq 5.0$ points for SF-36 subscales, $\geq 2.5$ points for SF-36 component summary scores, and $\geq 4$ points for FACIT-Fatigue scores. The Mann-Whitney $U$ test was used for comparisons. Linear regression models were next used to adjust for possible confounding factors; these included age, sex, ethnic origin, disease activity and duration, organ damage, corticosteroid use and use of immunosuppressants.

Results Patients receiving AMA $(\mathrm{N}=1098)$ performed better than patients who did not receive AMA $(\mathrm{N}=586)$ with regard to SF-36 physical component summary score, physical functioning, role physical, bodily pain, FACIT-Fatigue scores, EQ5D score and EQ-5D visual analogue scale score. The difference in SF-36 physical functioning was the greatest one among the SF-36 parameters, exceeding the corresponding MCID. The association between AMA use and better physical functioning was still significant after adjustment for potential confounding factors $(\beta=0.08 ; \mathrm{P}=0.001)$. In this analysis, Asian patients performed better in physical functioning $(\beta=0.07$; $\mathrm{P}=0.004)$ while African/African American patients performed worse $(\beta=-0.07 ; P=0.003)$. High disease activity $(\beta=-0.09$; $\mathrm{P}<0.001)$ and organ damage $(\beta=-0.12 ; \mathrm{P}<0.001)$ were also independent factors of worse physical functioning, whereas corticosteroid use independently improved the outcome $(\beta=0.06 ; P=0.022)$.

Conclusions AMA use is associated with favourable physical functioning in patients with SLE, independently of other factors.

\section{P171 CARDIOVASCULAR RISK IN YOUNG PATIENTS WITH LUPUS: INVESTIGATING METABOLIC BIOMARKERS AND THE IMMUNOLOGICAL PHENOTYPE}

George Robinson, Kirsty Waddington, Leda Coelewij, Junjie Peng, Anna Radziszewska, Chris Wincup, Hannah Peckham, David Isenberg, Yiannis Ioannou, Ines Pineda-Torra, Coziana Ciurtin, Elizabeth Jury. Dept. of Medicine, University College London, London, UK
Background Cardiovascular disease is the leading cause of mortality in patients with Juvenile-onset Systemic Lupus Erythematosus (JSLE) not attributable to lupus flare. This study used a multi-omic approach to investigate cardiovascular risk (CVR) in JSLE patients.

Methods NMR-based serum metabolomic biomarker analysis (including 113 different lipoprotein measures assessing lipoprotein size and lipid content) was performed on a discovery cohort of JSLE patients $(n=31$, median age 19). Data was analysed using cluster, receiver operating characteristic (ROC) and logistic regression analysis. Results were validated in a second JSLE cohort $(n=31$, median age 19). Flow cytometry evaluated 28 immune cell subsets and RNAseq assessed gene expression in matched patient samples.

Results Unbiased hierarchical clustering of metabolomic data identified 2 JSLE patient groups, each with a complex and unique lipoprotein profile. Group-1 had decreased high density lipoproteins (HDL) and increased very low and low density lipoproteins (VLDL/LDL) and Group-2 had elevated HDL but reduced VLDL/LDL indicating an association with high and low CVR respectively. These groups were validated in a separate JSLE cohort and Apolipoprotein (Apo) B:A1 ratio was identified as a predictive and longitudinally stable biomarker of CVR (ROC area under the curve>0.99).

The high and low CVR groups were also associated with a unique immune phenotype characterised by altered correlations between $\mathrm{T}$ and $\mathrm{B}$-cell subsets and significantly increased $\mathrm{CD} 4+$ and reduced CD8 + T-cell subsets in high vs low CVR. Transcriptomic analysis of T-cell subsets identified 70 genes upregulated and 62 downregulated in High vs Low CVR patients and pathway analysis identified membrane sphingolipid metabolism and lipid-mediated signalling as the top regulated pathways associated with CVR. Additionally, genes previously related with increased CVR, including IFNG and NLRP2, were significantly increased in the high CVR group.

Conclusion Multiomic analysis identified a putative predictive biomarker (ApoB:A1 ratio) and novel immunopathogenic pathways associated with increased CVR in JSLE.

\section{P172 MAINZER LUPUS SCORE (MLS) A NEW QUESTIONNAIRE FOR LUPUS TREATMENT}

Jessica Hoschek, Matthias Dreher, Simone Boedecker, Julia Weinmann-Menke, Andreas Schwarting. Division of Rheumatology and Clinical Immunology, University Medical Center of the Johannes Gutenberg University Mainz, Mainz, Germany

\subsection{6/lupus-2020-eurolupus.214}

Background For the diagnosis and treatment of the systemic lupus erythematosus (SLE), numerous scores and indices already exist with different focal points, which are mostly extensive and time consuming. The MLS is a short newly developed questionnaire consisting out of four items to consider whether it is necessary to adjust current therapy: Occurrence of new lupus-specific symptoms, subjective measurement of quality of life (Qol), change of serological abnormalities and the occurrence of organ damage accrual.

Methods Patients who had at least 3 visits since 2016 in our clinic were able to participate. Spearman-correlation was used to correlate MLS and several other well established scores for disease activity in SLE (SLEDAI, ECLAM, SLICC). Additionally, scores for subjective assessment of mental and physical state were used (FSMC, BDI, WAI) to obtain a preliminary 
impression applicability and expressiveness. Remission is defined as target in therapy of SLE. According to SLEDAI and current treatment recommendations patients were divided in four groups of disease activity (Remission, Remission on treatment, LLDAS, active disease).

Results It was possible to include 72 SLE patients with 305 visits in the study. $64(88 \%)$ of them were female and 8 patients were male. Average age was $48(\mathrm{SD} \pm 13.1)$ and the media duration of lupus disease was 15.6 years (SD \pm 8.7). There was a significant correlation between MLS and ECLAM $(p<0.001)$, WAI $(p=0.027)$ and BDI $(p=0.003)$, whereas the SLEDAI and cSLEDAI just show correlation with BDI $(p=0.008)$, respectively $(p=0.042)$. Additionally, a correlation between MLS and the remission status was found $(\mathrm{p}<0.001)$.

Conclusions The MLS is a 10 minute easy to administer questionnaire in clinical routine. To be highlighted is that the MLS considers subjective health parameters, like Qol which might be relevant for disease treatment. Further results will be presented at the conference.

\section{P173 IS USE OF HYDROXYCHLOROQUINE ASSOCIATED WITH BETTER PATIENT REPORTED OUTCOMES IN LUPUS?}

Meenakshi Jolly, Vinay Sehgal. Rheumatology, Rush University, Chicago, USA

\subsection{6/lupus-2020-eurolupus.215}

Background Use of Hydroxychloroquine (HCQ) is known to be associated with less disease activity, flares, damage and better survival in patients with Systemic Lupus Erythematosus (SLE). It is not known if its use is also associated with better patient reported health outcomes, a core outcome in SLE.

Methods International data from Study on Outcomes of Lupus (SOUL) on LupusPRO, a patient reported disease targeted Quality of life (QOL) tool, from 2,157 patients with SLE, were compared based on use HCQ. Disease activity and damage were assessed using SELENA-SLEDAI and SLICC-ACR/ SDI. T tests and Chi square tests were used for comparisons. Linear regression analyses were undertaken with summary LupusPRO health (HRQOL), non-health (NHRQOL) and Lupus Impact Tracker (LIT) scores as the dependent variables, and HCQ as the independent variable. LIT scores were derived from LupusPRO data. Similar analysis for damage was also conducted.

Results Mean age was $40.5 \pm 12.8$ years. Ninety-three percent were women. HCQ use 798/2157 (37\%) was inversely associated with age, Asian race, disease duration, lupus nephritis, neurological and hematologic manifestations, and damage. HCQ use was directly associated with presence of photosensitivity. On univariate analysis, HCQ use was associated with better QOL ( $\beta$ 6.19, 95\% CI 4.15, 8.24, P $\leq 0.001$ for LupusPRO-HRQOL) and less impact on daily life ( $\beta-9.37,95 \%$
CI $-12.24,-6.50, \mathrm{P} \leq 0.001$ for LIT). Other predictors for QOL and LIT were age, education, Asian race and disease activity. On multivariate analyses (adjusted for age, education, Asian status) use of HCQ was independently associated with better outcomes (LupusPRO-HRQOL and LIT). However, addition of disease activity variable to the models resulted in loss of independent association of HCQ use with better outcomes, suggesting mediation through disease activity (table 1). Similarly, mediation was seen for beneficial effects of HCQ use on damage through disease activity.

Conclusions HCQ use in SLE is associated with better health outcomes (LupusPRO-HRQOL, impact on daily life, damage), and the effects are mediated through disease activity modification.

Acknowledgements Behalf of SOUL group.

\section{P174 EXTENDED ARTERIAL ULTRASOUND REVEALING INCREASED INTIMA MEDIA THICKNESS AND RELATION TO IMPAIRED MICROCIRCULATION IN SYSTEMIC LUPUS ERYTHEMATOSUS}

1,2 Christina Svensson, ${ }^{3}$ Hanna Jonasson, ${ }^{3}$ Tomas Strömberg, ${ }^{4} \mathrm{Per}$ Eriksson, ${ }^{4}$ Christopher Sjöwall, ${ }^{1,2}$ Helene Zachrisson. 'Dept. of Clinical Physiology, Linköping University Hospital, Linköping; ${ }^{2}$ Dept. of Medical and Health Sciences, Linköping University, Linköping; ${ }^{3}$ Dept. of Biomedical Engineering, Linköping University, Linköping; ${ }^{4}$ Rheumatology/Division of Neuro and Inflammation Sciences, Dept. of Clinical and Experimental Medicine, Linköping University, Linköping, Sweden

\subsection{6/lupus-2020-eurolupus.216}

Background Systemic lupus erythematosus (SLE) is a chronic inflammatory disease characterized by multiple organ involvement. Atherosclerosis is the underlying cause of SLE-related cardiovascular disease. With high frequency ultrasound it is possible to differ between atherosclerosis and inflammatory findings in the vessel wall. Our hypothesis is that both macroand microcirculation are impaired in SLE.

Methods Sixty patients (52 women, 8 men), range 23-63 years, classified with SLE according to the 2012 SLICC criteria, and 60 healthy controls (52 women, 8 men), range 23-63 years, were investigated. Intima-media thickness (IMT) was recorded with high frequency ultrasound (GE Logic E9) in common carotid arteries (CCA), common femoral arteries (CFA) and the aortic arch. Microcirculatory oxygen saturation was assessed with EPOS (Enhanced Perfusion and Oxygen Saturation) (PeriFlux 6000, Perimed, Järfälla, Sweden). The EPOS system measures red blood cell tissue fraction, speed resolved perfusion and oxygen saturation in the microcirculation of the skin.

Results IMT in common carotid artery (CCA) was $0.56 \pm 0.10$ $\mathrm{mm}$ in SLE patients vs $0.54 \pm 0.13 \mathrm{~mm}$ in healthy controls (ns). IMT in common femoral artery (CFA) was $0.58 \pm 0.24$ $\mathrm{mm}$ in SLE patients vs $0.48 \pm 0.12 \mathrm{~mm}$ in healthy controls

Abstract P173 Table 1 Mediation of effects of HCQ use with outcomes through disease activity

\begin{tabular}{|c|c|c|c|c|c|c|c|c|c|c|c|c|}
\hline \multicolumn{7}{|c|}{ LIT } & \multicolumn{6}{|c|}{ LupusPRO-HRQOL } \\
\hline & \multicolumn{3}{|c|}{ Univariate } & \multicolumn{3}{|c|}{ Multivariate } & \multicolumn{3}{|c|}{ Univariate } & \multicolumn{3}{|c|}{ Multivariate } \\
\hline Variable & B & $95 \% \mathrm{Cl}$ & p-value & B & $95 \% \mathrm{Cl}$ & p-value & B & $95 \% \mathrm{Cl}$ & p-value & B & $95 \% \mathrm{Cl}$ & p-value \\
\hline Activity (SLEDAI) & 2.040 & $0.90,3.18$ & $\leq 0.001$ & 2.830 & $1.25,4.40$ & $\leq 0.001$ & -1.84 & $-2.70,-0.97$ & $\leq 0.001$ & -2.29 & $-3.59,-0.98$ & 0.001 \\
\hline $\mathrm{HCQ}$ & -9.370 & $-12.24,-6.50$ & $\leq 0.001$ & -3.050 & $-7.93,1.84$ & 0.221 & 6.190 & $4.15,8.24$ & $\leq 0.001$ & 3.640 & $-0.46,7.73$ & 0.080 \\
\hline
\end{tabular}

